\title{
Wavelet-analysis of the non-stationary signal of fluorescence of an ensemble of point scatterers
}

\author{
Sergey Bozhokin*, and Igor Sokolov \\ Peter the Great St. Petersburg Polytechnic University, 195251 St. Petersburg, Russia
}

\begin{abstract}
On the basis of quantum microscopic approach we calculate dynamics of fluorescence of a cold atomic ensemble confined to special atomic trap and excited by pulse radiation. Using continuous wavelet transform with adaptive Morlet wavelet we analyze spectral composition of the fluorescence and its modification with time after the exciting pulse is switched off. Dependence of the spectrum on direction of fluorescence and its polarization is also analyzed.
\end{abstract}

The purpose of this work is a theoretical analysis of the dynamics of the secondary emission of atomic ensembles cooled to sub-Doppler temperatures in special traps and excited by pulsed radiation.

Atomic clouds in traps are well described by a model of motionless point scatterers and allow a consistent quantum microscopic description based on solution of the non-stationary Schrödinger equation [1-3] for a joint system consisting of atoms and an electromagnetic field. Based on this approach we calculate the time dependence of the field strength of an electromagnetic wave emitted by the considered atomic assemblies. The main attention is focused on the analysis of the spectral composition of this radiation and its changes with time after the excitation is swished off.

For the spectral analysis of non-stationary signals, a number of approaches are currently used. To select the best of them, we compared the two most effective - the Gabor window conversion and the continuous wavelet transform (CWT) [4-6]. This comparison was carried out on the basis of calculation of Pearson's correlation coefficients for model signals with known time and spectral properties. The comparison showed some advantages of the CWT method, especially when using the adaptive Morlet wavelet (AMW) [6] which contains a control parameter $m$ that allows, if necessary, to increase either the temporal or the spectral resolution.

All specific calculations have been made on the basis of CWT using AMW for atomic clouds having a spherical shape with a random but uniform on average distribution of atoms. Averaging was carried out from this random distribution. The radius of the cloud was $R=45 \lambda$, the density of atoms $n_{0}$ was $n_{0} \hbar^{3}=0.002$ (here $\lambda=1 / k, k$ is the wave number of the incident radiation). The exciting pulse was assumed to be resonant and circularly polarized, its duration $\tau_{L}$ was chosen much less than the lifetime $\tau_{a}$ of the excited states of the atoms: $\tau_{L} / \tau_{a}=0.1$, which makes it possible to excite efficiently collective states in a

\footnotetext{
*Corresponding author: bsvjob@mail.ru
} 
wide spectral interval. Fluorescence signals in different directions were calculated for helicity-preserving $H \| H$ and helicity-nonpreserving $H \perp H$ polarization channels.

For all the cases considered, a complex time dependence of the investigated signals is obtained. The fluorescence intensity decreases in accordance with a multiexponential law. For the short times after the end of the exciting pulse the damping rate exceeds the rate of spontaneous decay of free atoms. Superradiance is observed. For long times, the main contribution is made by long-lived collective states, and the sub-radiation effect takes place. When calculating the spectral composition under conditions of a significant attenuation of the fluorescence intensity, we analyze the signals normalized to the instantaneous value of their amplitude.

Fig. 1 illustrates the spectrum of radiation at $\theta=\pi / 20$ (with respect to propagation direction of the incident wave) for one polarization channel. Fig. 1a shows the CWT $|V(v, t)|$ of the fluorescence signals, and Fig. $1 \mathrm{~b}$ demonstrates a frequency $v_{m}(t)$, which corresponds to the maximal value $|V(v, t)|$ for a given instant of time $t$. Fig. 1a corresponds to $m=50$, at which the spectral resolution is well determined. In Fig. 1b the AMW control parameter is $m=1$, and in this case the temporal modification of the spectra is determined in detail.
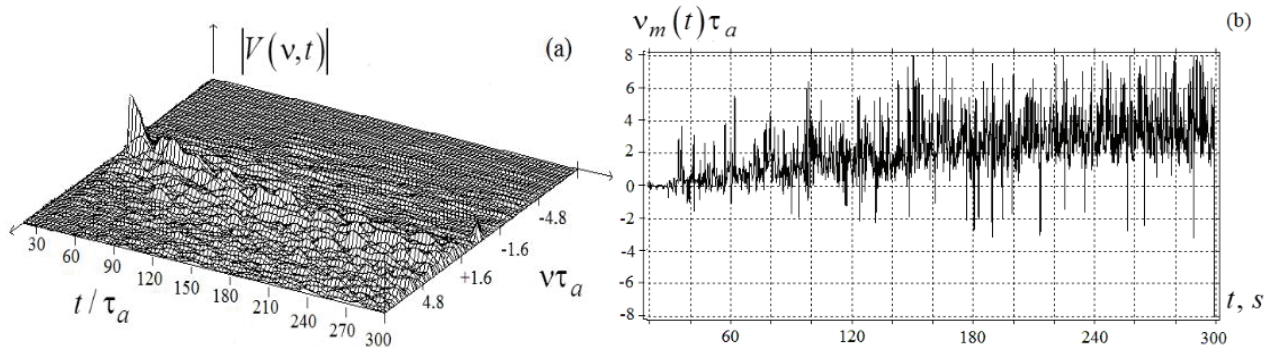

Fig. 1. CWT $|V(v, t)|$ and time dependence of frequency $v_{m}(t)$ for $H \perp H, \theta=\pi / 20$.

Figs. $1 \mathrm{a}$ and $1 \mathrm{~b}$ show essential difference between the emission spectra of a cloud and the spectra of free atoms, even in the case of a relatively dilute medium. These features of the spectrum are due to the presence of randomly formed pairs of closely located atoms. Such pairs form a kind of quasimolecule, the parameters of the excited states of which differ substantially from the parameters of atomic states by the energies and by the lifetimes. The contributions of these quasimolecules become most noticeable at large times $t \gg \tau_{a}$ after the end of the excitation, when the monatomic excitations have already completely relaxed.

Our calculations revealed also a significant difference among the spectra for different polarization channels as well as for different directions of fluorescence. These differences were explained by the features of the energetic spectra of the diatomic clusters.

This work was supported by the Russian Science Foundation (project No 17-12-01085).

\section{References}

[1] I.M. Sokolov, D.V. Kupriyanov, M.D. Havey, J. Exp. Theor. Phys. 112, 246 (2011)

[2] Ya.A. Fofanov, A.S. Kuraptsev, I.M. Sokolov et al., Phys. Rev. A 87, 063839 (2013)

[3] A.S. Kuraptsev, I.M. Sokolov, Phys. Rev. A 90, 012511 (2014)

[4] S.A. Mallat, Wavelet tour of Signal Processing (NY, Academic Press, 2008)

[5] C.K. Chui, An Introduction to Wavelet (NY, Academic Press, 1992)

[6] S.V. Bozhokin, S.V. Zharko, N.V. Larionov et al., Tech. Phys. 62, 837 (2017) 